\title{
Evaluación de la categorización de las urgencias gineco-obstétricas en un hospital del sur de Chile
}

\author{
Mercedes Carrasco Portiño, PhD 1,2,3, Daniela Godoy Novoa ${ }^{a}$, Alexis González-Burboa,

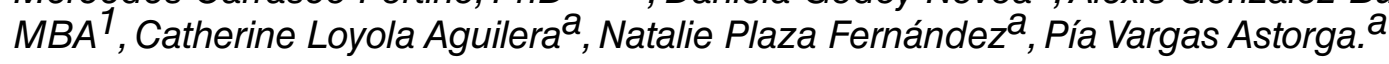 \\ ${ }^{1}$ Departamento de Obstetricia y Puericultura, Facultad de Medicina, Universidad de Concepción, Chile. ${ }^{2}$ Grupo de \\ investigación de Salud Pública, Universidad de Alicante, España. ${ }^{3}$ Observatorio de Políticas Públicas y Salud, Universidad \\ de Alicante, España.
}

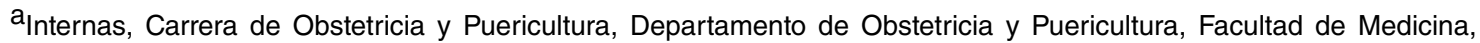
Universidad de Concepción, Chile.
}

\section{RESUMEN}

Objetivo: Caracterizar las consultas de urgencias gineco-obstétricas de un Hospital del sur de Chile en el año 2013. Métodos: Estudio descriptivo de corte transversal retrospectivo. Se analizaron los registros de la urgencia gineco-obstétrica (UGO) durante el primer semestre del 2013 que ascendieron a 4.898 consultas. Resultados: Un $85 \%(n=4.155)$ de las consultas atendidas en UGO fueron pacientes que concurrieron directamente desde su domicilio. El $67,9 \%(n=3.495)$ de las atenciones en la UGO se otorgaron en horario diurno promediando 815 consultas por mes. Los principales motivos de consulta fueron otras patologías asociadas al embarazo tales como enfermedades respiratorias agudas, transgresión alimentaria, alergias, hiperémesis gravídica, molestias mecánicas $(19,5 \%, n=897)$, el trabajo de parto y el parto $(7,4 \%, n=342)$ y la vulvovaginitis $(6,5 \%, n=302)$. El $65 \%(n=2.835)$ del total de las urgencias gineco-obstétricas con datos completos fueron bien categorizadas según el protocolo de priorización del hospital de estudio. Un $96,6 \%$ $(n=167)$ de las pacientes no requirió trasladó a un centro hospitalario de mayor complejidad. Conclusiones: Existe una sobre-utilización de la red de urgencia gineco-obstétrica por parte de las usuarias, colapsando el nivel terciario de atención con patologías que podrían ser resueltas en el nivel primario. Desde la matronería se debería liderar la instauración de un instrumento específico de TRIAGE gineco-obstétrico, como también la educación a la comunidad sobre uso adecuado de la red asistencial de nuestro país.

\section{PALABRAS CLAVE: TRIAGE, servicio médico de urgencia, obstetricia y ginecología}

\section{SUMMARY}

Objective: To characterize obstetric and gynecological consultations at the emergency department of a hospital in southern Chile in 2013. Methods: Cross-sectional descriptive retrospective study. Obstetric and gynecological emergency department records (OGE) were analyzed during the first half of 2013 which amounted to 4,898 . Results: $85 \%(n=4155)$ of the consultations assisted at OGE were patients who attended directly from home. $67.9 \%(n=3,495)$ of attentions at the OGE were developed in daytime averaging 815 visits per month. The main reasons of consultation were other diseases of pregnancy such as acute respiratory diseases, food transgression, allergies, hyperemesis gravidarum, mechanical discomforts $(19.5 \%, n=897)$, labor and delivery $(7.4 \%, n=342)$ and vulvovaginitis $(6.5 \%, n=302) .65 \%(n=2835)$ of all gynecological obstetric emergencies with complete data were well categorized according to protocol prioritization studied hospital. A $96.6 \%$ of the patients did not require transfer to a high complexity hospital. 
Conclusions: There is an over-utilization of the obstetric and gynecological emergency network by users, collapsing the tertiary level of care with diseases that could be resolved at the primary level. The midwifery should lead the creation of a specific instrument TRIAGE obstetrics and gynecology, as well as community education on proper use of the healthcare network in our country.

\section{KEYWORD: TRIAGE, emergency medical service, obstetric and gynecologic}

\section{INTRODUCCIÓN}

La red de urgencia del sistema público de salud chileno, en cuyos centros de mayor complejidad se sitúa la Urgencia Gineco-Obstétrica (UGO), vive sobre demandada por un gran número de patologías de baja complejidad (1). La UGO atiende a toda mujer que concurre espontáneamente o es derivada del nivel secundario por el Policlínico de Alto Riesgo Obstétrico (ARO) o de establecimientos de Atención Primaria de Salud (APS).

Para priorizar las consultas espontáneas la evidencia respalda el uso del TRIAGE $(2,3)$, herramienta que en el ámbito gineco-obstétrico ha demostrado utilidad para reducir la mortalidad materna en los hospitales (4), categorizar embarazadas sin controles prenatales (5) y para la práctica de la oncología ginecológica $(6,7,8)$, entre otras. Además, señala para su ejecución al matrón/a, profesional experto en salud sexual y reproductiva e idóneo para priorizar la atención médica especializada (9).

En Chile, la producción científica sobre el tema es escasa, aunque existen publicaciones, principalmente en pediatría, que reportan el uso del TRIAGE en las urgencias $(10,11)$, y también es posible encontrar documentos ministeriales $(12,13)$ y protocolos de categorización en las UGO de algunos hospitales (14).

El objetivo de este estudio es caracterizar las consultas de UGO de un Hospital del sur de Chile durante el primer semestre del año 2013.

\section{MATERIAL Y MÉTODOS}

Estudio descriptivo de corte transversal retrospectivo, realizado en usuarias que consultaron en el servicio de Urgencia Gineco-Obstétrica (UGO) del Hospital San José de Coronel, $8^{\mathrm{a}}$ Región, Chile, durante el primer semestre del año $2013(n=4.898)$.

Los criterios de inclusión fueron el universo de consultas del servicio de urgencia del Hospital en estudio, en la UGO, durante el primer semestre del año 2013. Los criterios de exclusión fueron los datos incompletos en el registro hospitalario.

Los datos para el análisis de los motivos de consulta, se obtuvo desde los registros utilizados en el servicio de urgencia del Hospital de estudio. Las variables seleccionadas para el estudio se agruparon en las siguientes categorías: tipo de ingreso a la UGO, previsión de salud, mes, día, hora, comuna de residencia, centro de salud de origen, lugar de procedencia, diagnóstico, categorización (Clasificación en hospital de estudio: Categoría 1 (C1): Paciente con riesgo vital inmediato; requiere resucitación por tener alteradas sus funciones vitales. Ejemplo: Paro cardiorespiratorio; Categoría 2 (C2): Paciente en situación de emergencia; requiere acción en un tiempo breve por tener sus signos vitales alterados o algún signo sugerente de enfermedad grave o deterioro rápido. Ejemplo: Metrorragia moderada; Categoría 3 (C3): Paciente en situación de urgencia; presenta estabilidad hemodinámica pero riesgo potencial de deterioro en el tiempo. Ejemplo: Mastitis aguda; Categoría 4 (C4): Paciente con problema de salud poco urgente; no presenta riesgo vital. Ejemplo: Embarazada con rash alérgico; Categoría 5 (C5): Paciente con afección crónica y aguda pero no urgente; no requiere de una acción diagnóstica o terapéutica en el momento. Ejemplo: Amenorrea (12), tipo de atención, profesional que otorga la atención, lugar de referencia, traslado a otro centro.

$\mathrm{La}$ información fue registrada en una planilla Excel que reunía los datos estadísticos de las usuarias utilizados en el servicio de UGO. Para el análisis de los datos se utilizó el programa SPSS 18.0.

Este estudio cuenta con la aprobación del Comité ético-científico de la Facultad de Medicina de la Universidad de Concepción.

\section{RESULTADOS}

Se identificaron 14 personas menores de 10 años y el resto $(n=4.872)$ presentó una media de edad de 29 años (rango 10 - 90 años).

En relación a la comuna de residencia de las usuarias, podemos destacar que el 93,9\% $(n=4.576)$ pertenecía a la misma comuna donde se encuentra el hospital de estudio y el 6,9\% $(n=334)$ restante de las usuarias provenía de comunas aledañas.

El $34,8 \% \quad(n=1.698)$ estaba inscrita en el policlínico adosado del hospital, mientras el 55,7\% ( $n=2.714$ ) lo estaba en Centros de Salud Familiar (CESFAM) de misma comuna donde está ubicado el hospital de estudio, y $9,9 \% \quad(n=480)$ estaba 
vinculada a otros centros o se atendía de forma particular.

En relación a la procedencia, el $85,3 \%(n=4.155)$ de las consultas atendidas en UGO correspondieron a usuarias que concurrieron directamente desde su domicilio, un $3,9 \%(n=188)$ fue derivada del Policlínico de ARO, un 5,4\% $(n=262)$ de la Urgencia de Adultos y un $5,9 \%(n=287)$ fueron referidas de otras comunas aledañas.

En relación a la previsión de las usuarias consultantes, el $97,8 \%(n=4768)$ pertenecía a FONASA (Fondo Nacional de Salud)y de este grupo el $75,1 \%(n=3.660)$ correspondía a los tramos A y $B$. Cabe señalar que del resto de las consultas un $0,2 \%(n=11)$ fueron realizadas por usuarias beneficiarias de ISAPRE (Instituciones de Salud Previsional) y un $1,0 \%(n=49)$ por beneficiarias de DIPRECA (Dirección de Previsión de Carabineros de Chile) o PRAIS (Programa de Reparación y Ayuda Integral en Salud y Derechos).

La Tabla I muestra que durante el período de estudio un $71,7 \%(n=3.495)$ de las atenciones en la
UGO se otorgaron en horario diurno promediando 815 consultas por mes. En relación a los días de consulta, de lunes a viernes la tendencia se mantuvo en torno al $15,2 \%$, disminuyendo el fin de semana a un promedio de $11,9 \%$ de las consultas. En este sentido, destaca que las consultas en urgencias de toda la semana rondan el $70 \%$ en las categorías $\mathrm{C} 4$ y $\mathrm{C} 5$, es decir, de menor complejidad, siendo la más alta el domingo con un $74,1 \%$.

Por otro lado, respecto a los meses del año involucrados, destaca que las consultas de complejidad variable (C3) o baja (C4 y 5) descendieron en el mes de enero, pero no así las consultas de alta complejidad (C2) o emergencia vital (C1).

La Tabla II muestra que los principales motivos de consulta durante el período en estudio fueron otras patologías del embarazo tales como enfermedades respiratorias agudas, transgresión alimentaria, alergias, hiperémesis gravídica, molestias mecánicas $(18,4 \%, n=897)$, el trabajo de parto y el parto $(7,0 \%, n=342)$ y la vulvovaginitis $(6,2 \%$, $\mathrm{n}=302)$.

\section{Tabla I \\ FRECUENCIA DE CONSULTAS EN URGENCIA GINECO-OBSTÉTRICA DEL HOSPITAL SAN JOSÉ DE CORONEL, SEGÚN HORARIO, MESES Y DÍAS, PRIMER SEMESTRE 2013}

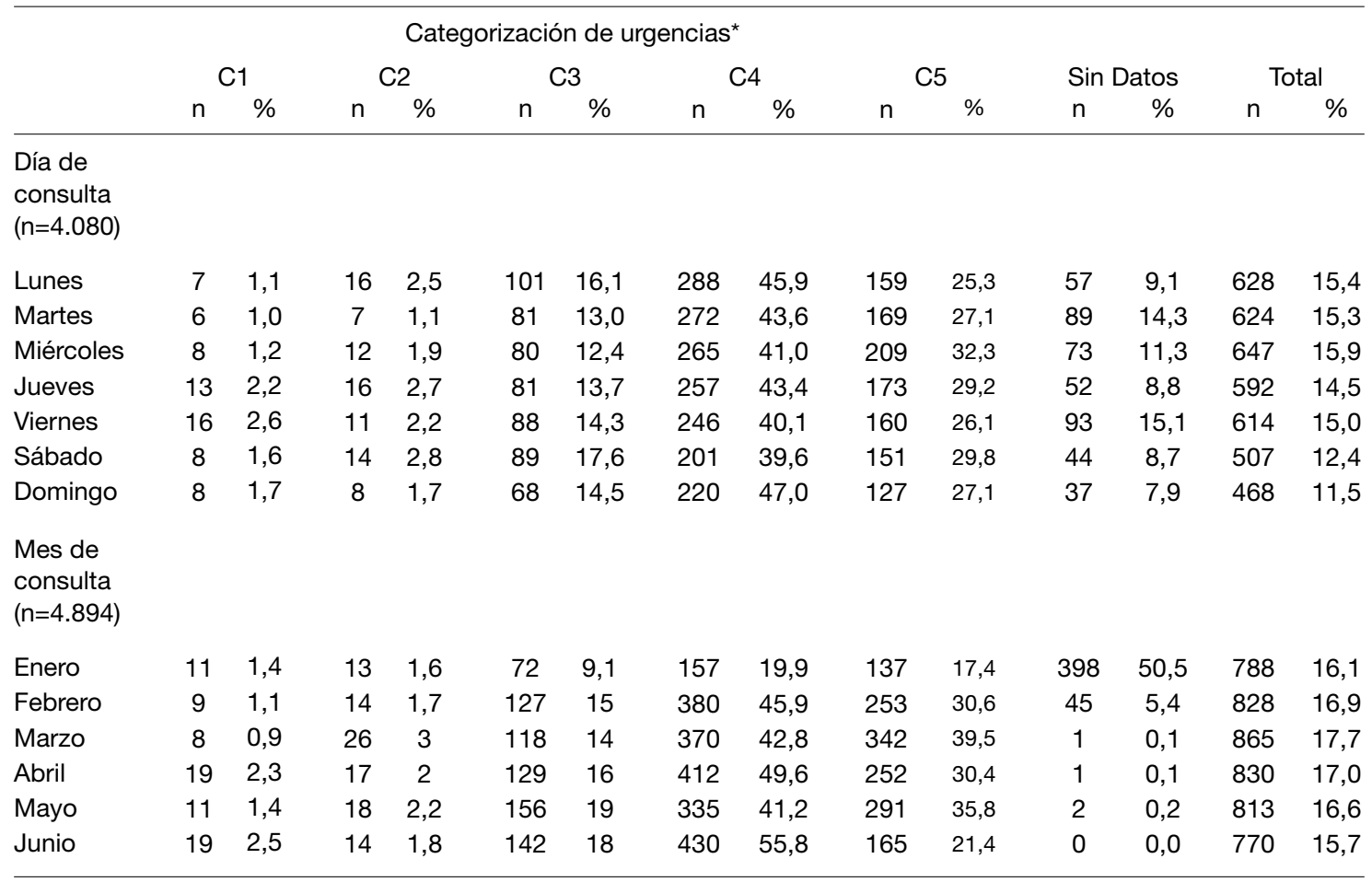

${ }^{*}$ C1: emergencia vital, requiere atención inmediata; C2: emergencia evidente, alta complejidad en su patología; C3: patología de complejidad variable; C4: requiere procedimiento diagnóstico o terapéutico de baja complejidad; C5: consulta general de baja complejidad. 


\section{Tabla II \\ DISTRIBUCIÓN DE LAS CONSULTAS SEGÚN DIAGNÓSTICO DE INGRESO A LA UNIDAD DE URGENCIAS GINECO-OBSTÉTRICAS DEL HOSPITAL SAN JOSÉ DE CORONEL, PRIMER SEMESTRE 2013}

\begin{tabular}{|c|c|c|}
\hline Patología & $\mathrm{n}$ & $\%$ \\
\hline \multicolumn{3}{|l|}{ Ginecología: } \\
\hline${ }^{\star \star}$ Alteraciones mamarias (mastitis, mastalgia, galactorrea, abscesos, nódulos) & 199 & 4,3 \\
\hline ^^Ginecológico otros (prolapso, mioma, endometrio patológico, pólipo, etc.) & 156 & 3,4 \\
\hline $\begin{array}{l}{ }^{\star \star D} \text { Disfunción menstrual (amenorrea, dismenorrea, hipermenorrea, hipomenorrea, oligo/ } \\
\text { polimenorrea) }\end{array}$ & 147 & 3,2 \\
\hline Hemorragia de origen ginecológico & 138 & 3,0 \\
\hline *^Absceso Bartholino, Flegmón vulvar, foliculitis púbica, várices vulvares. & 73 & 1,6 \\
\hline **Algia pélvica & 276 & 6,0 \\
\hline Evaluación ginecológica & 248 & 5,4 \\
\hline${ }^{\star \star D}$ DIU: descendido o incrustado & 56 & 1,2 \\
\hline Extracción de cuerpos extraños & 3 & 0,1 \\
\hline **Anticoncepción de emergencia & 11 & 0,2 \\
\hline **Infecciones de transmisión sexual & 11 & 0,2 \\
\hline \multicolumn{3}{|l|}{ Obstetricia: } \\
\hline $\begin{array}{l}{ }^{\star *} \text { Embarazo otros (enfermedades respiratorias agudas, transgresión alimentaria, alergias, } \\
\text { hiperémesis gravídicas, molestias mecánicas, etc.) }\end{array}$ & 897 & 19,5 \\
\hline Diabetes gestacional / diabetes mellitus descompensadas & 26 & 0,6 \\
\hline Síntoma/trabajo de parto prematuro & 29 & 0,6 \\
\hline Taquicardia fetal/hipoactividad fetal & 8 & 0,2 \\
\hline Óbito fetal & 3 & 0,1 \\
\hline Trabajo de parto (fase latente o activa)/parto & 342 & 7,4 \\
\hline Aborto (amenaza, síntoma, en evolución, completo, retenido, séptico) & 266 & 5,8 \\
\hline **Diagnóstico de embarazo (test) & 256 & 5,6 \\
\hline $\begin{array}{l}\text { Embarazo con comorbilidad asociada (crisis epiléptica, crisis asmática, lumbalgia crónica, } \\
\text { etc.) }\end{array}$ & 59 & 1,3 \\
\hline Embarazo ectópico, molar & 52 & 1,1 \\
\hline Síndrome hipertensivo del embarazo & 49 & 1,1 \\
\hline Traumatismos embarazada/ginecológicos & 48 & 1,0 \\
\hline Metrorragia (I, II, III trimestre ) & 41 & 0,9 \\
\hline \multicolumn{3}{|l|}{ Otros: } \\
\hline Herida operatoria/episiorrafia infectada y/o dehiscencia & 81 & 1,8 \\
\hline **Usuaria no espera atención & 58 & 1,3 \\
\hline Constatación de lesiones & 36 & 0,8 \\
\hline *^Puérpera: constipación, rash alérgico, estado gripal, molestias generales. & 38 & 0,8 \\
\hline${ }^{\star \star}$ Curación & 34 & 0,7 \\
\hline${ }^{\star \star}$ Infecciones del tracto urinario & 185 & 4,0 \\
\hline${ }^{\star \star}$ Referida para hospitalización & 281 & 6,1 \\
\hline${ }^{\star}$ Total & 4.610 & 100 \\
\hline
\end{tabular}

*Del total de consultas efectuadas, que corresponden a 4.892, se encontraron sin datos 282 usuarias, las que corresponden al $5,7 \%$.* Patologías de baja complejidad. 
La Figura 1 muestra la categorización de las usuarias que consultaron en UGO durante el período en estudio. Cabe destacar que existe un porcentaje de $9,2 \%(n=446)$ de atenciones que no fueron categorizadas.

La Tabla III destaca que el porcentaje de usuarias bien categorizadas según protocolo de priorización de UGO del Hospital de estudio, correspondió a 58,2\% $(n=2.835)$ del total. Se excluyeron de este cálculo las usuarias sin datos.

En cuanto a los profesionales que otorgaron las prestaciones, un $89,7 \%(n=4.368)$ de las usuarias fueron atendidas por el equipo constituido por Matrona-Médico-Técnico en Enfermería (TENS), un $6,1 \%(n=296)$ por Matrona y TENS, un $4,5 \%$ $(n=217)$ por Matrona y Médico y un $0,2 \%(n=10)$ fue atendido solamente por Matrona.

Tras la atención, un $69,9 \% \quad(n=3.407)$ de las usuarias fueron derivadas a su domicilio y/o CESFAM de procedencia, mientras que un $29,9 \%$ $(n=1.460)$ requirió recibir prestaciones de mayor complejidad. Un $0,8 \%(n=39)$ fue referida a otros centros de comunas aledañas. Un 96,6\% ( $n=4.717)$ de las usuarias consultantes no requirió traslado a un centro de mayor complejidad.

\section{DISCUSIÓN}

En el presente estudio, la mayor parte de las consultantes en la UGO consultaron en forma espontánea provenientes de su domicilio, predominando las consultas diurnas con una leve tendencia a disminuir los fines de semana. La mayor parte de las consultantes residían en la comuna en donde se localiza el centro asistencial en estudio por tanto pertenecían a establecimientos de APS de la misma; solo un pequeño porcentaje de las consultantes en la UGO se atendían en el sector privado. Se observó que la mayor parte de las consultas que se atendieron durante el período en estudio fueron patologías de baja complejidad, en su mayoría atendidas por equipo multidisciplinario y derivadas a su domicilio tras la atención.

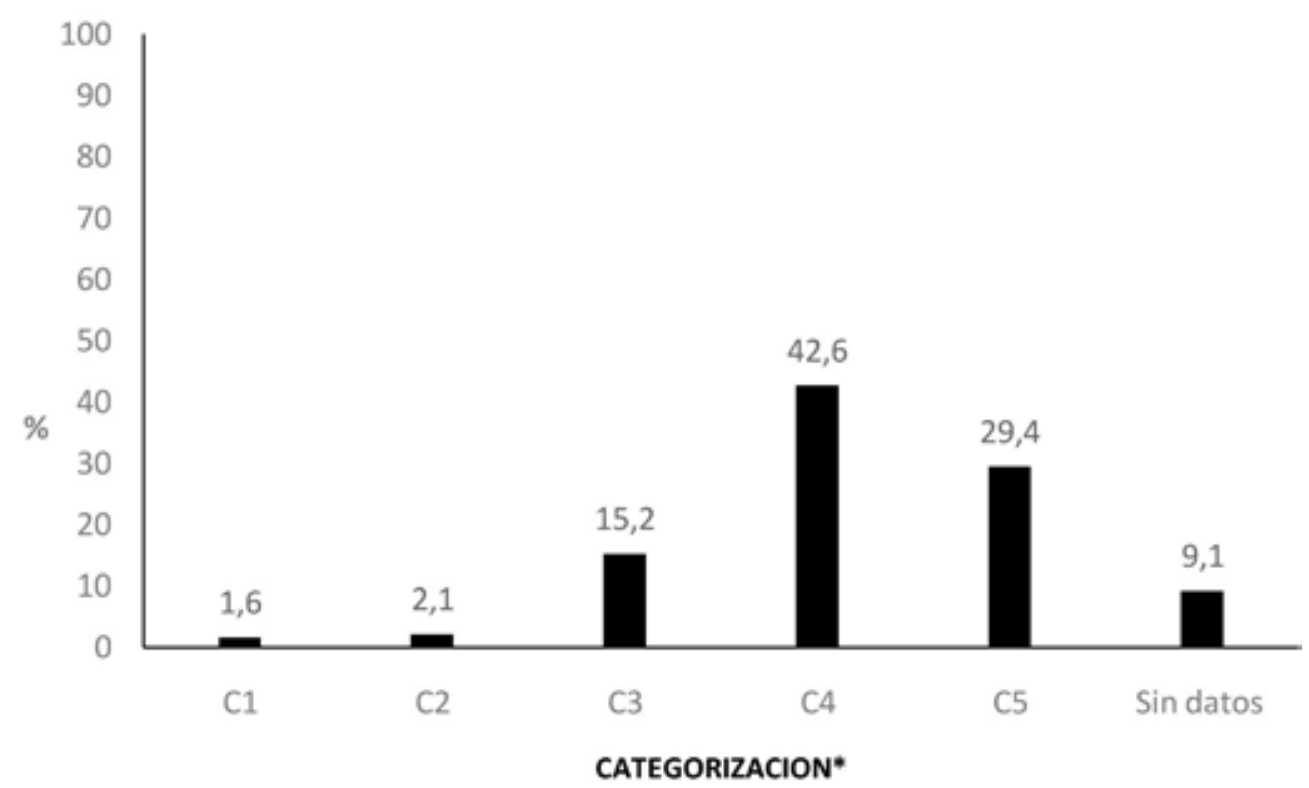

Figura 1. Categorización según nivel de complejidad de atención en la Unidad de Urgencias Gíneco-Obstétricas del Hospital San José de Coronel, primer semestre de 2013(n=4.892). *C1: emergencia vital, requiere atención inmediata; C2: emergencia evidente, alta complejidad en su patología; C3: patología de complejidad variable; $\mathrm{C} 4$ : requiere procedimiento diagnóstico o terapéutico de baja complejidad; C5: consulta general de baja complejidad. 
Tabla III

\section{DISTRIBUCION DE DIAGNÓSTICOS DE INGRESO A LA UNIDAD DE URGENCIAS GINECO-OBSTÉTRICAS DEL HOSPITAL SAN JOSÉ DE CORONEL, SEGÚN CATEGORIZACIÓN TRIAGE, PRIMER SEMESTRE $2013(\mathrm{n}=4.892)$}

Diagnóstico

Categorización de urgencias*

Correctamente

Categorizadas

$\begin{array}{llllllll}\mathrm{C} 1 & \mathrm{C} 2 & \mathrm{C} 3 & \mathrm{C} 4 & \mathrm{C} 5 & 99^{\star} & \mathrm{n} & \%\end{array}$

Ginecología

Absceso Bartholino, flegmón vulvar, foliculitis púbica, várices vulvares

Algia pélvica

Alteraciones mamarias (mastitis, mastalgia, galactorrea, abscesos, nódulos)

Disfunción menstrual (amenorrea, dismenorrea, hipermenorrea, hipomenorrea, oligo/polimenorrea)

DIU: descendido o incrustado

Evaluación ginecológica

Extracción de cuerpos extraños

Ginecológico otros (prolapso, mioma, endometrio patológico, pólipo, etc.)

Hemorragia de origen ginecológico

Infecciones de transmisión sexual

Vulvovaginitis

Anticoncepción de emergencia

\section{Obstetricia}

Diabetes gestacional / diabetes mellitus descompensadas

Síndrome hipertensivo del embarazo

Síntoma/trabajo de parto prematuro

Taquicardia fetal/hipoactividad fetal

Trabajo de parto (fase latente o activa)/parto

Traumatismos embarazada/ginecológicos

Referida para hospitalización

Embarazo con comorbilidad asociada (crisis epiléptica, crisis asmática, lumbalgia crónica, etc.)

Puérpera: constipación, rash alérgico, estado gripal, abdomen agudo, molestias generales.

Diagnóstico de embarazo (test)

Embarazo ectópico, molar

Embarazo otros (enfermedades respiratorias agudas, transgresión alimentaria, alergias, hiperémesis gravídicas, molestias mecánicas, etc.)

Metrorragia (I, II, III trimestre )

Óbito fetal

Pródromos

\begin{tabular}{|c|c|c|c|c|c|c|c|c|}
\hline 0 & 2 & 2 & 25 & 41 & 3 & 41 & 56,2 & 73 \\
\hline 1 & 2 & 20 & 178 & 66 & 9 & 66 & 23,9 & 276 \\
\hline 0 & 1 & 11 & 75 & 93 & 19 & 86 & 43,2 & 199 \\
\hline 0 & 3 & 18 & 27 & 99 & 0 & 99 & 67,7 & 147 \\
\hline 0 & 0 & 5 & 14 & 31 & 6 & 45 & 80,3 & 56 \\
\hline 2 & 1 & 14 & 53 & 135 & 42 & 135 & 54,6 & 247 \\
\hline 0 & 0 & 0 & 1 & 2 & 0 & 3 & 100 & 3 \\
\hline 1 & 4 & 14 & 60 & 51 & 26 & 111 & 70,5 & 156 \\
\hline 3 & 4 & 38 & 32 & 53 & 8 & 77 & 55,8 & 138 \\
\hline 1 & 1 & 1 & 1 & 7 & 0 & 7 & 63,6 & 11 \\
\hline 0 & 0 & 9 & 83 & 204 & 7 & 287 & 95,3 & 301 \\
\hline 0 & 0 & 0 & 2 & 9 & 0 & 11 & 100 & 11 \\
\hline 0 & 1 & 1 & 13 & 8 & 3 & 1 & 3,8 & 26 \\
\hline 9 & 3 & 15 & 14 & 2 & 6 & 12 & 24,4 & 49 \\
\hline 1 & 7 & 12 & 7 & 1 & 1 & 8 & 27,5 & 29 \\
\hline 0 & 0 & 2 & 4 & 2 & 0 & 0 & 0 & 8 \\
\hline 19 & 27 & 177 & 67 & 18 & 34 & 223 & 65,2 & 342 \\
\hline 2 & 3 & 7 & 24 & 6 & 6 & 5 & 10,4 & 48 \\
\hline 0 & 2 & 41 & 196 & 25 & 16 & 196 & $70 \%$ & 280 \\
\hline 2 & 2 & 8 & 27 & 2 & 18 & 59 & 199 & 59 \\
\hline 1 & 1 & 2 & 15 & 16 & 3 & 31 & 81,5 & 38 \\
\hline 0 & 0 & 19 & 80 & 125 & 32 & 125 & 48,8 & 256 \\
\hline 1 & 3 & 17 & 19 & 6 & 6 & 21 & 40,3 & 52 \\
\hline 6 & 7 & 64 & 581 & 163 & 75 & 581 & 64,8 & 896 \\
\hline 3 & 7 & 18 & 9 & 0 & 4 & 37 & 90,2 & 41 \\
\hline 2 & 0 & 0 & 1 & 0 & 0 & 2 & 66,6 & 3 \\
\hline 2 & 4 & 84 & 74 & 22 & 15 & 74 & 36,8 & 201 \\
\hline 1 & 0 & 3 & 4 & 39 & 11 & 11 & 18,9 & 58 \\
\hline
\end{tabular}

Otros

Usuaria no espera atención 
CONTINUACIÓN TABLA III

\begin{tabular}{lccccccccc}
\hline Diagnóstico & \multicolumn{3}{c}{ Categorización de urgencias* } & \multicolumn{3}{c}{$\begin{array}{c}\text { Correctamente } \\
\text { Categorizadas }\end{array}$} \\
& C1 & C2 & C3 & C4 & C5 & $99^{*}$ & $\mathrm{n}$ & $\%$ & Total \\
\hline Constatación de lesiones & 11 & 0 & 3 & 8 & 10 & 4 & 11 & 30,6 & 36 \\
Curación & 0 & 0 & 1 & 15 & 18 & 0 & 18 & 52,9 & 34 \\
Herida operatoria/episiorrafia infectada y/o dehis- & 0 & 2 & 6 & 34 & 30 & 9 & 40 & 49,3 & 81 \\
cencia & & & & & & & & & \\
Infecciones del tracto urinario & 0 & 1 & 7 & 100 & 54 & 23 & 108 & 58,3 & 185 \\
Sin datos & 4 & 4 & 38 & 126 & 76 & 36 & - & - & 284 \\
\hline Total & 77 & 102 & 743 & 2084 & 1438 & 447 & 2835 & 61,5 & 4892 \\
\hline
\end{tabular}

*Sin categorización

La mayoría de las consultantes del servicio de urgencia pertenecía a la misma comuna donde se asienta el establecimiento en estudio, tal como en otro estudio pero sobre consultas de urgencias infantiles (15), sin embargo, existe literatura que señala que las urgencias de los centros de mayor complejidad también son más susceptibles de ser consultadas por usuarios provenientes de otras comunas (16). Este trabajo aporta nueva información que señala, además, que la mayoría de las consultantes se encontraba inscrita en un CESFAM de la misma comuna del hospital.

En relación a la procedencia de las consultas, los hallazgos de este estudio concuerdan con literatura que reporta que la mayoría de las consultas fueron realizadas por usuarias que venían desde sus hogares, quienes consultaban por iniciativa propia sin pasar previamente por un nivel de menor complejidad $(17,18)$.

Teniendo en cuenta que la mayoría de las pacientes eran usuarias de FONASA, es decir, del seguro de salud del sistema público, se concuerda con otros estudios sobre urgencias (19) o consulta pediátrica (20), coincidiendo, además, en que corresponde a población de estrato socioeconómico vulnerable (21).

En cuanto a los patrones horarios, el número de consultas no presentó grandes variaciones dentro de los días de la semana ni durante los meses del estudio, privilegiándose el horario nocturno y evidenciando solo una leve baja en las consultas los fines de semana. Esto difiere de los datos de un estudio que encontró que la mayor cantidad de consultas se realizaron en horario diurno preferentemente el día lunes, mostrando la mayor demanda de atención en los meses de febrero y marzo (22).

Un número considerable de las pacientes que acudieron a la UGO estaban inscritas en establecimientos de atención primaria, donde podrían haber resuelto gran parte de las consultas, pudiendo evidenciar esto, baja resolutividad en el nivel primario de atención (23). Esto podría deberse al escaso conocimiento de la ciudadanía respecto al correcto uso de la red pública de salud, problemas de acceso a los servicios y/o descoordinación entre los distintos niveles de atención (24).

La mayoría de las patologías atendidas en la UGO no eran urgentes, por tanto esto justifica que un amplio porcentaje de las consultantes hayan sido referidas a sus hogares, sin requerir traslado ni prestaciones especializadas. Los hallazgos mostraron además que, tras la aplicación del TRIAGE, existían patologías que fueron clasificadas en más de una categoría. Esto manifiesta la existencia de una relativa subjetividad al aplicar este instrumento. Por tanto, y para evitar las variaciones de la atención en salud y asegurar la calidad de ésta, es necesario contrastar la práctica clínica con los estándares de referencia (25).

Como limitación de este estudio se puede señalar la dificultad de analizar otros factores que puedan influir en la consulta de UGO al trabajar con datos secundarios. Esto podría ser una limitante al momento de contrastar y entender mejor fenómenos como los patrones de consulta de las pacientes.

\section{CONCLUSIONES}

Se puede afirmar que existe sobre-utilización de la red de urgencia, específicamente de la urgencia gineco-obstétrica por parte de las usuarias del sistema de salud quienes colapsan el nivel terciario de atención con patologías que podrían ser resultas en el nivel primario. Sin embargo, como profesionales de la matronería debemos liderar el proceso de la educación de la comunidad en la utilización de los servicios de atención en salud, tanto primaria, 
secundaria o de alta complejidad, así como también la validación de un TRIAGE específico para el área gineco-obstétrica para las urgencias de nuestros centros hospitalarios, cuestión que requiere un trabajo conjunto con profesionales médicos especialistas en esta área de competencia.

\section{REFERENCIAS}

1. Situación de los sistemas de atención de urgencia en Chile: propuestas técnicas para mejorar su gestión, 2012. Comisión de Urgencias del Colegio Médico de Chile. Hallado en: http://www.mdu.cl/wp-content/ uploads/2014/08/INFORME-FINAL-URGENCIA.pdf. Acceso el 15 de diciembre de 2015.

2. Modelo de Gestión de Establecimientos Hospitalarios, 2008. Subsecretaría de Redes Asistenciales, Ministerio de Salud. Hallado en: http://www.hjnc.cl/docs/ Modelo_de_gestion_de_Establecimientos_Hosp.pdf. Acceso el 15 de diciembre de 2015.

3. Manual para la implementación de un sistema de TRIAJE para los cuartos de urgencia, 2010. Organización Panamericana de la Salud. Hallado en: http:// new.paho.org/hq/dmdocuments/2011/HSS_IS_Manual_Sistema_Tiraje_CuartosUrgencias2011.pdf. Acceso el 15 de diciembre de 2015.

4. Alvarado R, Arroyo M, Hernández $C$, Vélez M, Márquez P. Manejo del TRIAGE Obstétrico y Código Mater en el Estado de México. Enferm Univ 2012;9(2):6171.

5. Knight E, Morris M, Heaman M. A descriptive study of women presenting to an obstetric triage unit with no prenatal care. J Obstet Gynaecol Can 2014;36(3):21622.

6. Vercellini P, Cribiù F, Del Gobbo A, Carcangiu M, Somigliana E, Bòsari S. The oncofetal protein IMP3: a novel biomarker and triage tool for premalignant atypical endometriotic lesions. Fertil Steril 2013;99(7):1974-9.

7. Enakpene C, Omigbodun A, Goecke T, Odukogbe A, Beckmann M. Preoperative evaluation and triage of women with suspicious adnexal masses using risk of malignancy index. J Obstet Gynaecol Res 2009;35(1):131-8.

8. Alcázar J, Royo P, Jurado M, Mínguez J, GarcíaManero M, Laparte C, et al. Triage for surgical management of ovarian tumors in asymptomatic women: assessment of an ultrasound-based scoring system. Ultrasound Obstet Gynecol 2008; 32(2):220-5.

9. Obregón-Gutiérrez N, Rebollo-Garriga G, Costa Gil MP, Calle-Del Fresno S, Puig-Calsina S. El TRIAJE Obstétrico: nuevo reto para las matronas. Experiencia del Hospital Parc Taulí en la implementación de un sistema de Triaje Obstétrico. Matronas Prof 2011;12(2):49-53.

10. Medina J, Ghezzi C, Figueredo D, León D, Rojas G, Cáceres L, et al. Triage: Experiencia en un Servicio de Urgencias Pediátricas. Rev Chil Pediatr 2007;78(2):211-6.

11. Galaz C, Valladares $Y$, Sánchez G, De la Fuente $M$, Yentzen G. Triage pediátrico: un sistema efectivo de priorización en los servicios de urgencia. Rev Chil Pediatr 2005;76(1):25-33.
12. Norma técnica $N^{\circ} 149$ sobre Estándares de información en salud - Estándar de atención de urgencia de los niveles de alta, mediana y baja complejidad y de la atención primaria, 2013. Ministerio de Salud. Hallado en: http://www.deis.cl/wp-content/uploads/2013/07/ EST\%C3\%81NDAR-DE-ATENCI\%C3\%93N-DE-URGENCIA.pdf. Acceso el 15 de diciembre de 2015.

13. Manual operativo: Servicios de atención primaria de urgencia de alta resolución (SAR), 2015. Subsecretaría de Redes Asistenciales. Hallado en: http://www. tecmed.cl/web/images/2015_marzo/doc_PDF.pdf. Acceso el 15 de diciembre de 2015.

14. Priorización en la atención de urgencia obstétrica y ginecológica, 2012. Complejo hospitalario San José. Hallado en: http://www.ssmn.cl/transparencia/ CHSJ2010/juridica/Resolucion_4077_\%202012.pdf. Acceso el 15 de diciembre de 2015.

15. Leiva $H$, Ortega $M$, Martinovic $C$. Perfil de la consulta de urgencia infantil en el Hospital Regional Rancagua 2002-2003. Rev Chil Salud Pública. 2004;8(2):72-7.

16. Fodor A, Navarrete E, Cáceres E, Muñoz F. Atención de Urgencia Odontológica en el Hospital de Urgencia Asistencia Pública. Rev Dent Chile 2005;96(3):3-6.

17. Espinel $M$, Romero $M$, Fernández $L$, Torres $J$ y D'antonio S. Utilización por Cuenta Propia de los Servicios de Urgencias Hospitalarias: Razones que dan las Personas con Problemas de Salud de Baja Complejidad para Utilizar estos Servicios. Pol y Soc 2011;48(2):329-52.

18. Reinoso S, Díaz G, Robles A, Pereiro E, Fernández E, López A.Forma de acceso a los Servicios de Urgencia hospitalarios y pertinencia de la consulta. Rev Clín Med Fam 2011;4(3):205-10.

19. Font I, Izquierdo, Puiguriguer J. Análisis de las consultas a un servicio de urgencias hospitalario según el origen de los pacientes. Emergencias 2009;21: 262268.

20. Méndez B, Herrera P, Guerra H, Dattas J, Muñoz B, Velasco J. Estructura de la consulta pediátrica en el Servicio de Urgencia: Hospital Infantil Roberto del Río. Rev Chil Pediatr2005;76(3):259-65.

21. Miranda C, Martínez F, Fariña J, Mihovilovic C. Consultas en Servicio de Urgencia Hospital Roberto del Río: ¿Cuáles son las razones de esta preferencia por sobre el nivel primario de atención? Rev Ped 2007;4(2).Hallado en: http://www.revistapediatria.cl/ vol4num2/pdf/2_Consultas_SUHRR.pdf. Acceso el 06 de enero de 2016.

22. Martín G, Cáceres J. Un Método de obtención del patrón estacional de frecuentación de un servicio de urgencias hospitalario. Rev Esp Salud Pública2005;79(1):5-15.

23. Román O. Resolución clínica en la atención primaria de salud. Rev Méd Chile 2011;139(9):1240-1.

24. Pujol E, Gené J, Sans M, Sampietro-Colom L, Pasarín $M$, Iglesias-Pérez $B$, et al. El producto de la atención primaria definido por profesionales y usuarios. Gac Sanit 2006;20(3):209-219.

25. Ortells N, Paguina M. Indicadores de calidad y seguridad del paciente en la enfermería de urgencias: un valor seguro. Enferm Glob 2012;11(26):184-90. 